this is a preprint, the final version is published on Applied Surface science: https://authors.elsevier.com/a/1cjbdcXa\%7Eyt-u

Applied Surface Science, 552 (2021) 149454

https://doi.org/10.1016/j .apsusc. 2021.149454

\title{
Kinetic Monte Carlo simulations of the diffusion and shape evolution of single-layer clusters on a hexagonal lattice with and without external force
}

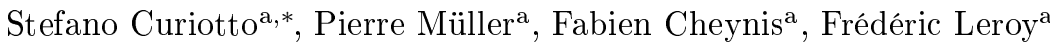 \\ ${ }^{a}$ Aix Marseille Univ, CNRS, CINAM, Marseille, France
}

\begin{abstract}
In this work we investigate the diffusion of $2 \mathrm{D}$ surface islands and voids as a result of atomic diffusion on terraces, by kinetic Monte Carlo simulations based on a hexagonal lattice. We show that the diffusion coefficient of small clusters strongly depends on their size and does not follow a monotonic law. In particular, the diffusion coefficient of small voids increases with the cluster size, reaches a plateau and then decreases. We also study the displacement velocity of clusters when a force biases the probability of the atomic jump direction. We show how the cluster velocity depends on the force and on the cluster size. According to the force direction and amplitude, the shapes of the clusters can be strongly modified.
\end{abstract}

\section{Introduction}

Surface diffusion plays a key role in catalysis, adsorption, growth, reactivity, as it affects the stability and the morphology of material surfaces by means of step rearrangement [1] and cluster diffusion 22. Surface diffusion is the most common surface transport process, so that recently it has been proposed to use it to control the motion of nano-objects on a surface [3]. This would open new perspectives in nanofabrication. In this context, understanding cluster diffusion on various surfaces is becoming a new challenge. Clusters predominantly migrate by the individual motion of atoms instead of collective motion. In absence of any external force, the motion of the clusters is random (Brownian), whereas in presence of an external force, as for instance electromigration [4-7, a bias is added to the random motion and thus enables to control the cluster motion. If cluster diffusion is now well documented by experiments and analytical approach (at least for large islands) [8], Kinetic Monte Carlo (KMC) simulations have brought new perspectives especially for studying small clusters for which atomic configurations play an important role [9, 10]. However, most of the studies have been performed on square lattices. In this article we use KMC simulations to study the diffusion of singlelayer clusters (2D voids and $2 \mathrm{D}$ islands) on a hexagonal (or triangular) lattice in presence or in absence of an external force like electromigration. We show that most of the results obtained on square lattices are still valid on hexagonal lattices, even if subtle changes are evidenced.

\footnotetext{
*Corresponding author

Email addresses: curiotto@cinam.univ-mrs.fr (Stefano Curiotto), muller@cinam.univ-mrs.fr (Pierre Müller), cheynis@cinam. univ-mrs.fr (Fabien Cheynis)

leroy@cinam.univ-mrs.fr (Frédéric Leroy)
}

For the largest clusters, simple scaling-laws expressed in terms of size-dependent diffusion coefficient do not depend on the lattice symmetry. For small clusters, the shapes and shape fluctuations play a major role, leading to the appearance of finite-sizes effects that may differ on square or hexagonal lattices. Our goal in this article is to give a clear description of the mechanisms of cluster diffusion on hexagonal lattices with or without an external force. For this purpose, we start by describing some concepts of surface diffusion from a microscopic point of view, taking into consideration single atomic jumps with a special focus on how atomic jumps lead to cluster diffusion. We pay special attention to small clusters for which simple scaling laws are not valid, leading to specific behaviors for welldefined cluster sizes. The step-by-step study of the cluster motion in terms of single atomic jumps aims at providing a detailed description of the cluster diffusion properties and shape evolution. Section 2 details the model used in the simulation. Section 3 addresses the diffusion of clusters without the effect of external forces. We pay particular attention to small clusters, where discrete effects modify the behaviors predicted by continuous models. Section 4 shows how the clusters displace under a force, their velocity and shape for different temperatures and force values. In section 5 we change the symmetry of the lattice from 6 -fold to 3 -fold and we simulate the shapes of clusters with and without the effect of an external force. The most relevant findings are then summarized in the conclusions.

\section{Kinetic Monte Carlo model}

In our model, we consider a $2 \mathrm{D}$ hexagonal (or triangular) lattice of positions that can be either occupied by atoms or empty (see figure $1 \mathrm{p}$ ). The simulation box has at least 10000 lattice sites and periodic boundary conditions. 

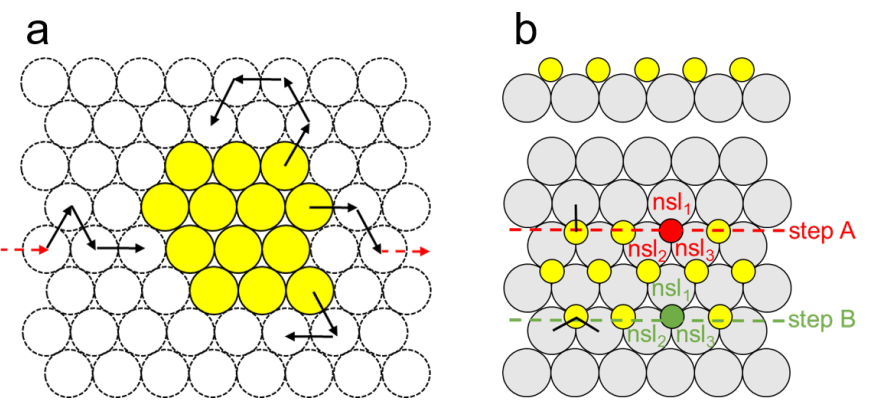

Figure 1: a: Schematics of lattice positions of a single plane that can be either empty (white) or occupied by atoms (yellow). Possible jumps are shown with arrows, the red dashed arrows represent jumps at the box edges with boundary conditions. b: side view and top view of atoms in our model. The small yellow circles represent atoms of the top layer, while large grey circles are atoms under the top layer. Atoms at steps A and B, like the red and green atoms, have the same number of neighbors but they have a different local arrangement (the red atom has only one neighbor in the bottom layer at the exterior of the step, while the green atom has two neighbors in the bottom layer, see the short black lines). The atoms under the top layer that are neighbors of the red and the green atoms are indexed nsl1, nsl2 and nsl3 (see appendix A).

Atoms can jump from an occupied to an empty neighbor position and cannot jump on top of already occupied positions, therefore atomic jumps are not allowed on top of islands and outside voids (infinite Ehrlich-Schwoebel barrier).

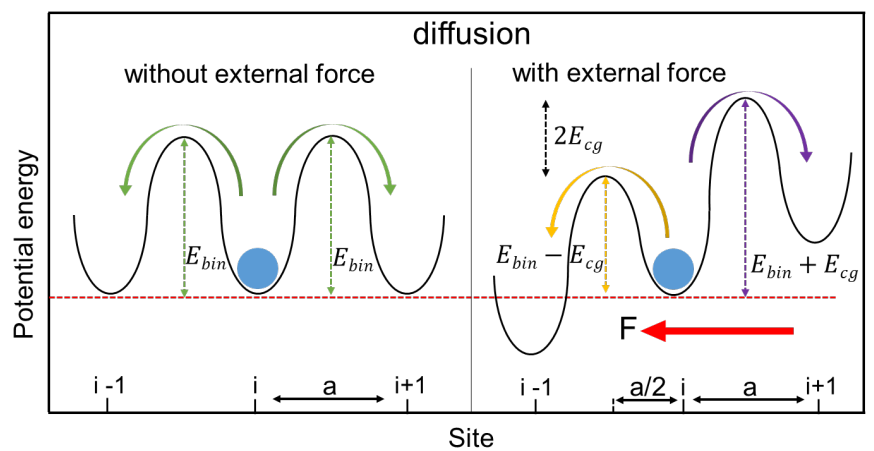

Figure 2: Schematics of the energy barrier that an isolated adatom has to overcome to jump from a site $i$ to a nearby position $i+1$ or $i-1$. With a force (red horizontal arrow, right panel), the jump energy is no more symmetric in the two directions: it is lower for a jump towards the left (yellow curved arrow), in the force direction, while it is higher for a jump towards the right (violet curved arrow). A jump in the force direction is thus favored.

The model is based on a previous version [11]. The jump probability depends only on the energy of the departing site (Arrhenius dynamics), i.e. on the binding energy of the jumping adatom $\left(E_{b i n}\right.$, see appendix $\left.A\right)$ and on the external force acting on it (see the schematics of figure 22. The jump rates are proportional to:

$$
\exp \left(-\frac{E_{b i n}+E_{c g}}{k T}\right)
$$

$k$ is the Boltzmann constant and $\mathrm{T}$ is the temperature.
$E_{c g}$ is the energy change due to the external force. It is positive when the jump direction is against the force and negative when the jump is helped by the force. We have used the expression of $E_{c g}$ given in [5], but modified for a hexagonal lattice:

$$
E_{c g}=|F| \cdot \cos \left(\frac{\pi}{3} \cdot b-\delta\right) \cdot \frac{a}{2}
$$

which takes into account the angle between the force and the atomic jumps. $|F|$ is the external force acting on an atomic jump; $a$ is the lattice separation between two neighbor positions, we take it equal to 1 in all directions; $b$ is an integer that defines the arrival site in anticlockwise order, with values between 1 and $6 ; \delta$ is the angle between the force and the $\mathrm{x}$ axis. Atomic jumps are selected according to a rejection-free Kinetic Monte Carlo algorithm. The time needed for an atomic jump is equal to the inverse of the sum of all jump rates [12.

The results of section 5 are obtained with an improved version of the model, modified to represent also the (111) surface plane of cubic structures, where atomic steps can form either 100 or 111 microfacets (A and B steps in the following) with the underlying plane, as shown in figure 13. B steps are more stable than A steps [13, 14]. It is not possible to reproduce this energy difference taking into account only the number of bonds of surface atoms with in-plane neighbors. In order to consider the step energy difference in our simple lattice model, we have used a binding energy that depends on the bonds formed by surface atoms with the subsurface atomic layer, that is considered fully occupied, according to a procedure detailed in appendix A. This model is general for $2 \mathrm{D}$ triangular lattices and illustrates qualitatively different scenarios of homo-epitaxial cluster diffusion biased by a force. It was originally developed to understand the diffusion of $2 \mathrm{D} \mathrm{Si}$ islands on $\mathrm{Si}(111)$ (see some experimental results in [15]), but the energies and temperatures used are not characteristic of a particular system. The model could be adapted to different systems by changing the binding energies (see appendix A).

\section{Cluster diffusion}

The model can be used to simulate random diffusion when the force $|F|$ is set to 0 . The simulations of diffusion of single adatoms and vacancies, available in the supplementary material, allow to validate the model. In the following we focus on the diffusion of clusters.

\subsection{Large clusters}

$2 \mathrm{D}$ voids and islands are clusters of vacancies and adatoms respectively. Their edges fluctuate with time, because atoms can detach and re-attach somewhere else. The diffusion of clusters on a surface has been investigated by different authors experimentally (some prominent examples are 16-19) and theoretically. As 

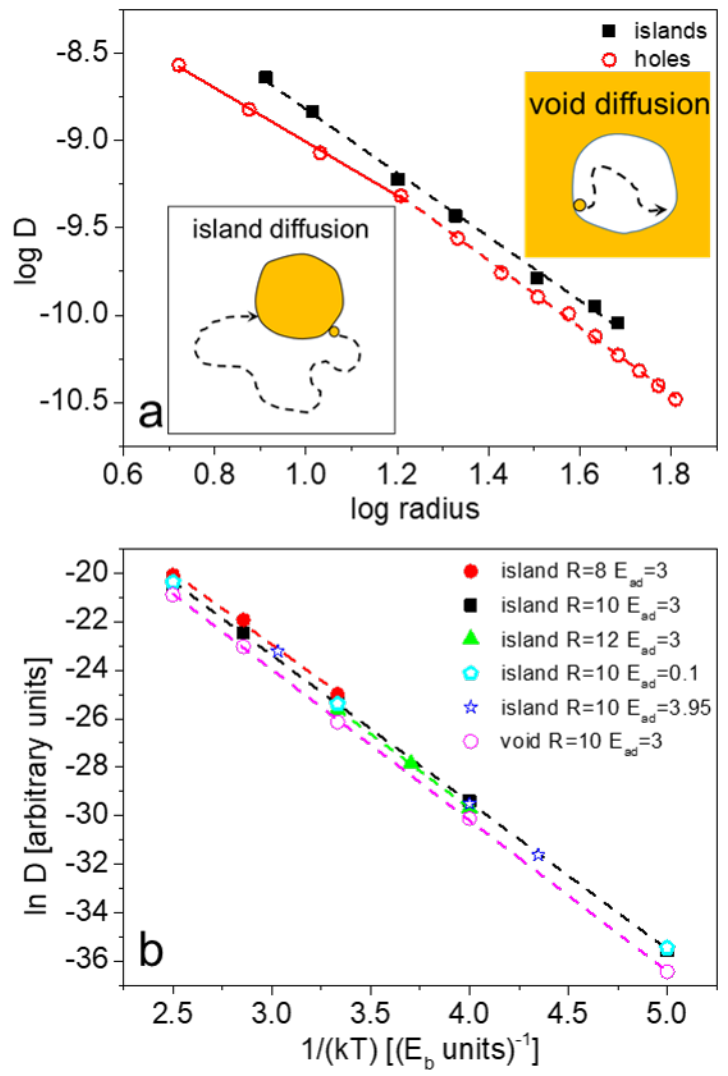

Figure 3: a: Diffusivity of islands (black square) and voids (red empty circles) as a function of their radius, in logarithmic scale $\left(\mathrm{kT}=0.4 E_{b}\right)$. b: Logarithm of the cluster diffusion coefficient as a function of $1 /(\mathrm{kT})$ for different islands and a void, as defined in the figure. The simulations of cluster diffusion are carried out with $k_{n s l}=0$ (see appendix A). Simulations with different $E_{a d}$ have been carried out to evaluate its effect on $D_{C}$.

a consequence of atom diffusion, the edges of islands and voids are modified and the cluster center of mass displaces. Three different mechanisms of cluster motion are usually considered: atomic diffusion at the cluster periphery, diffusion on the terrace outside and inside clusters, and uncorrelated detachment/attachment of atoms (evaporation-condensation). Simple scaling arguments lead to the conclusion that diffusion should depend on the cluster radius $R: D_{C} \propto R^{-\alpha}$, with $\alpha=1$, 2 or 3 for kinetics limited by attachment/detachment, terrace diffusion and periphery diffusion respectively [17, 20. Khare and Einstein [8, 21] used an analytical continuous description of the cluster boundary to describe the diffusion of clusters, confirming the above finding. Several authors, the first of them being Voter [2, also considered the discrete nature of clusters, to take into account the effect of kinks and facets, and performed Monte Carlo simulations to investigate cluster diffusion. Most of them focused mainly on the periphery diffusion and evaporation-condensation mechanisms [9, 10, 22, 28], finding some discrepancies from the power laws detailed above. Cluster diffusion mediated by atoms diffusing on the terrace has been less studied, but Scanning Tunneling Microscopy [17] and KMC simulations on a rectangular lattice to describe the $\mathrm{Ag}(110)$ surface [29] confirm the power law and $\alpha=2$.

In order to get further insights into the diffusion of clusters on a surface with atoms organized in a compact hexagonal pattern, we have simulated the evolution of clusters when atoms detach from the perimeter, diffuse on the terrace and re-attach to the cluster. Notice that periphery diffusion is not impeded. We have verified that the cluster average square displacement is proportional to the time, so that we can define the cluster diffusion coefficient $D_{C}=\frac{\left\langle r_{\text {cluster }}^{2}\right\rangle}{4 t}$. The number of atomic jumps of each simulation ranges between $10^{8}$ (cluster with $\mathrm{R}=5$ ) and $10^{10}$ $(\mathrm{R}=45)$. The convergence of the $D_{C}$ measurement is verified using the procedure detailed in the supplementary material, where control charts are shown.

The black squares in figure 3 a show the diffusion coefficient of large islands for different radii in log-log scale ( $\mathrm{R}$ larger than 8 units, more than 200 atoms in the clusters). A linear fit through the points gives the value of $\alpha=1.9 \pm 0.1$, close to 2 . Diffusion of adatoms on the terrace controls the cluster diffusion. Islands are surrounded by a large terrace (the box size is much larger than that of the island) where adatoms can diffuse for a long time before re-attaching, therefore the kinetics for island displacement in that case are limited mainly by terrace diffusion.

For voids, we find that $\alpha$ is different for small ( $\alpha=$ $1.54 \pm 0.04)$ and large $(\alpha=1.92 \pm 0.02)$ voids (empty circles in figure 3a). As suggested by Sholl et al. 24, in small voids the diffusion time of an adatom between a detachment and an attachment event is small. The kinetics for the displacement of the cluster depends thus also on the time needed to detach an atom and not only on the time of adatom diffusion inside the void. The detachment effect contributes to $\alpha$ that is thus between 1 and 2. At lower temperatures, the exponent $\alpha$ further decreases because the detachment rates decrease more than the adatom diffusion rate. For voids we find $\alpha=1.54,1.45$ and 1.3 at $\mathrm{kT}=0.4,0.3$ and 0.25 respectively.

Voids displace slower than islands, but the diffusion difference decreases when the cluster size increases. The reason of this is that the step edge of islands is convex and therefore presents more atoms in kink positions, easy to detach from the perimeter, than voids, where the step edge is concave. In other words, on average, atoms at islands edges are less bound than atoms at void edges. This difference between islands and voids is exacerbated for small clusters. The detachment time of atoms from the periphery of small voids is thus expected to be high, and further accentuates the importance of the detachment kinetics found for small voids.

We now test the expression for the cluster diffusion coefficient given by Van Siclen [20, developed for terrace diffusion or correlated attachment-detachment mechanisms 
(i.e. an atom detaching from the cluster edge has higher probability to re-attach in a position close to the detachment site):

$$
D_{C}=\frac{<r_{c l u s t e r}^{2}>}{4 t}=\frac{D_{a d} \cdot c \cdot \Omega^{2}}{\pi \cdot R^{2}}
$$

$\Omega$ is the unit cell area and $c$ the adatom concentration. In the simulations it is possible to measure the average square displacement and also the number of adatoms around an island or inside a void. We can thus measure an adatom concentration. The measured cluster diffusivity can be compared with that predicted by the above equation using the measured concentration. Table 1 compares some values of diffusivity for islands and voids. The above equation is based on a continuous model, while in our simulations the atomic jumps are discrete and the displacement of a cluster could depend on facet nucleation. However the agreement is reasonable for both voids and islands. The high error on the calculated $D_{C}$ is due to the error on the measured adatom concentration. Our equations are valid for diffusion only outside islands and inside voids. The expression for the diffusion coefficient given by Khare and Einstein [8] corresponds to that of Van Siclen multiplied by a factor 2 because they allow diffusion both outside and inside the clusters (for instance considering also diffusion of adatoms on top of the islands).

We verify if, in analogy with the diffusion of a single adatom, we can write $D_{C}=D_{C 0} \cdot \exp -\frac{E_{\text {cluster }}}{k T}$, where $D_{C 0}$ is a prefactor and $E_{\text {cluster }}$ an effective cluster diffusion energy. The black squares in figure 3 b show an example of cluster diffusion coefficient in natural logarithm scale as a function of $1 /(k T)$ for an island, while the empty circles represent the diffusivity of a void with 10 atomic units radius. For each set, the points are well aligned, and, using the expression above, a linear fit gives an effective energy of $E_{\text {cluster }}=6.06 \pm 0.05 E_{b}$ for islands and $6.22 \pm 0.05$ for voids. $E_{\text {cluster }}$ does not correspond to the adatom diffusion energy $\left(E_{a d}=3 E_{b}\right)$. We have changed the adatom diffusion energy independently from other binding energies to check if $E_{\text {cluster }}$ changes. As shown by the empty pentagons and stars in figure $3 \mathrm{p}$, decreasing (resp. increasing) $E_{a d}$, the cluster diffusivity $D_{C}$ increases (resp. decreases) only slightly, and the slope of the linear fit in figure $3 \mathrm{p}$, i.e. $E_{\text {cluster }}$, does not change. While cluster diffusion as a function of size $\left(D_{C} \propto R^{-2}\right)$ suggests the importance of the adatom diffusion on the terrace, the adatom diffusion energy $E_{a d}$ does not play a role in the cluster diffusion energy. These findings are consistent with those of Heinonen et al. [27, who simulated cluster diffusion on (100) lattices but without extensive discussions.

To understand $E_{\text {cluster }}$ we have to consider in equation 1 also the adatom concentration and not only $D_{a d}$. The concentration $c$ depends from the balance between atoms that detach from edges and adatoms diffusing and jumping towards an edge and attaching to it [30. In our simulations a cluster is at equilibrium with an adatom gas surrounding it and there is no other structure that can capture the atoms detached from the cluster. Therefore, at long times, the number of atoms detaching from the cluster equals that of atoms attaching to it. On average, the time necessary to detach an atom is equal to the time needed to have an attachment to the cluster boundary. Adatoms on the terrace attach to a cluster with a probability proportional to the adatom concentration, to the adatom diffusion coefficient and to the length of the cluster perimeter (that can be considered as a cross section). The attachment time, inversely proportional to the attachment probability is then

$$
t_{\text {attachment }} \propto \frac{1}{c \cdot D_{a d} \cdot 2 \pi R}
$$

The probability to detach an atom from the cluster boundary depends on the number of boundary atoms $N_{\text {boundary }}$ and on their binding energy (i.e. on their neighbors). $t_{\text {detachment }}$ is inversely proportional to this probability:

$$
t_{\text {detachment }} \propto \frac{1}{\sum_{j=1}^{5} N_{j} \cdot \exp \frac{-E_{j}}{k T}}
$$

where $\mathrm{j}$ is the number of in-plane neighbors, $E_{j}$ is the binding energy of an atom with $\mathrm{j}$ in-plane neighbors, $N_{j}$ is the number of atoms at the edge of the cluster with $\mathrm{j}$ neighbors (see appendix $\mathrm{A}$ and figure A.14 and $\sum_{j=1}^{5} N_{j}=$ $N_{\text {boundary. }}$ Writing

$$
\frac{\sum_{j=1}^{5} N_{j} \cdot \exp \frac{-E_{j}}{k T}}{N_{\text {boundary }}}=<\exp \frac{-E_{j}}{k T}>
$$

then

$$
t_{\text {detachment }} \propto 1 /\left(N_{\text {boundary }}<\exp \left(\frac{-E_{j}}{k T}\right)>\right)
$$

This expression cannot be directly handled because the average of the exponentials depends on the $N_{j}$, that depend on the size of the cluster and on the temperature. We thus re-write the expression using an effective binding energy $E_{b i n}$ for all the boundary atoms, that can be found considering the energy needed to remove an entire row of a cluster edge. If the average number of atoms of an edge is $N_{\text {edge }}$, and they are removed one after the other, $N_{\text {edge }}-1$ are kinks, while the last one is more weakly bound (atom adsorbed at a step). Therefore

$$
<E_{\text {bin }}>=\frac{E_{\text {kink }}\left(N_{\text {edge }}-1\right)+E_{\text {ad-step }}}{N_{\text {edge }}}
$$

We thus write

$$
t_{\text {detachment }} \propto \frac{1}{N_{\text {boundary }} \exp \left(\frac{-E_{\text {kink }}\left(N_{\text {edge }}-1\right)+E_{\text {ad-step }}}{N_{\text {edge }} \cdot k T}\right)}
$$

With $t_{\text {attachment }}=t_{\text {detachment }}$ and considering that $N_{\text {boundary }} \propto 2 \pi R$, we find

$$
c \propto \exp \frac{-E_{k i n k}}{k T} \cdot \exp \frac{E_{a d}}{k T} \cdot \exp \frac{E_{k i n k}-E_{a d-s t e p}}{N_{\text {edge }} \cdot k T}
$$




\begin{tabular}{|c|c|c|c|}
\hline cluster type & $\mathrm{kT}$ & simulated $D_{C}$ & calculated $D_{C}$ \\
\hline island & 0.45 & $8.6 \cdot 10^{-9} \pm 0.5 \cdot 10^{-9}$ & $6.6 \cdot 10^{-9} \pm 0.7 \cdot 10^{-9}$ \\
\hline island & 0.5 & $3.4 \cdot 10^{-8} \pm 0.3 \cdot 10^{-9}$ & $2.6 \cdot 10^{-8} \pm 0.4 \cdot 10^{-8}$ \\
\hline void & 0.45 & $1.5 \cdot 10^{-9} \pm 0.2 \cdot 10^{-9}$ & $1.8 \cdot 10^{-9} \pm 0.4 \cdot 10^{-9}$ \\
\hline void & 0.5 & $5.9 \cdot 10^{-9} \pm 0.2 \cdot 10^{-9}$ & $7.1 \cdot 10^{-9} \pm 0.4 \cdot 10^{-9}$ \\
\hline
\end{tabular}

Table 1: Comparison of measured(simulated) and calculated (equation 1 Van Siclen expression 20]) cluster diffusion coefficients, for islands $(\mathrm{R}=10)$ and voids $(\mathrm{R}=20)$. The calculated $D_{C}$ has an error due to the error on the simulated concentration.

Notice that this expression includes, in the denominator of the exponential, the dependence of the concentration on the cluster size $\left(N_{\text {edge }} \propto R\right)$, as predicted by the GibbsThomson equation. Substituting the expression above in equation 1, we obtain:

$$
D_{C} \propto \exp \frac{-E_{k i n k}}{k T} \cdot \exp \frac{E_{k i n k}-E_{a d-s t e p}}{N_{\text {edge }} \cdot k T}
$$

The argument of the second exponential term in the above expression is negligible with respect to the argument of the first one and thus $E_{\text {cluster }} \approx E_{k i n k}=6 E_{b}$ in our simulations. However, changing the cluster radius, we have found that $E_{\text {cluster }}$ depends on it, though very slightly, and increases when $R$ increases (For $2 \mathrm{D}$ islands $E_{\text {cluster }}=5.92$, 6.06 and $6.10 E_{b}$ for $R=8,10$ and 12 ). This is qualitatively in agreement with the effect of the second exponential term in equation 3. Another approach to discuss $E_{\text {cluster }}$ that does not imply an effective energy is detailed in appendix B.

\subsection{Finite-size effects: 2D islands}

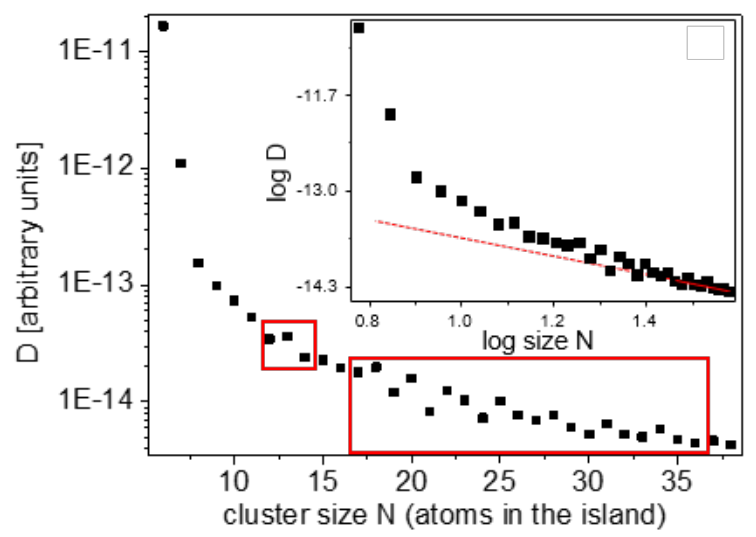

Figure 4: Cluster diffusion coefficient as a function of the number of atoms in simulations with $\mathrm{kT}=0.2$. The inset shows the same graph in $\log$-log scale to highlight that $D \propto N^{-\beta}$ holds only for large N.

Finite-size effects have been experimentally observed in nanoclusters of some systems. For instance, the catalytic properties of $\mathrm{Pd}$ nanoparticles show non-monotonic variations as a function of their size [31, 32]. Also the diffusion of small clusters can depend on their size in a non-trivial way, especially at low temperatures. As shown in the previous section, the diffusion coefficient decreases when the cluster size increases, and, for islands, it is proportional to $R^{-2}$. However, for small islands, this general trend changes. Figure 4 shows that $D_{C}$ decreases with the island size N, i.e. the starting number of atoms in the cluster. For large clusters, $\mathrm{N}$ is $\propto R^{2}$, therefore $D_{C} \propto R^{-2} \propto N^{-1}$; for small clusters $R$ is not significant, however we verify if the $D_{C}$ proportionality still holds. The inset of figure 4 shows that $\log D_{C}$ is proportional to $-1.2( \pm 0.3) \log N$ only for islands larger than about $\mathrm{N}=29$. For smaller sizes $D_{C}$ decreases faster than $N^{-1}$ and ceases to follow a size dependent power law. Figure 4 also shows that $D_{C}$ does not decrease steadily when $\mathrm{N}$ increases, but is punctuated by oscillations.

On square lattices, different authors have found that cluster diffusion does not follow a monotonic descending trend with the cluster size [9, 27, 33-35]. This is due to compact or "perfect size" clusters that are slower than others because their boundary is only constituted by strongly bound atoms (with few kinks and no adatoms at steps). The reasons of the oscillations are explained for (100) surfaces by Lai et al. [9]: clusters with perfect size, without adatoms at steps, diffuse slowly because the formation of a new edge allowing the cluster displacement requires a nucleation energy equivalent to the detachment of two kink atoms.

On a hexagonal lattice, clusters with symmetric perfect size are for instance those with $\mathrm{N}=7, \mathrm{~N}=19$ and $\mathrm{N}=37$, as shown in figure 5 . Those configurations have the minimum number of border atoms with the maximum possible inplane neighbors. They have 6 kinks, no adatoms at steps, and thus minimize the cluster step energy. However, other sizes (for instance $\mathrm{N}=10,12,13,14,16$ and for all clusters with $\mathrm{N} \geq 18$ ) also exhibit shapes with 6 kinks and no adatoms at steps (see two examples in figures 5 for $\mathrm{N}=12$ and $\mathrm{N}=13$ ). To understand the $D_{C}$ oscillations, we consider that a significant displacement of a cluster requires the detachment and re-attachment of atoms, formation of a new edge with at least two atoms. For instance, starting from a cluster with $\mathrm{N}=19$ shown in figure 5 after the removal of a first kink (blue in figure 5), two other kinks are created (green in figure 5), and all the boundary atoms still have at least 3 in-plane neighbors. Therefore the formation of a new edge requires at least detachment of two kinks. For $\mathrm{N}=20$, the low energy configuration has 4 kinks that are coupled: removing one of them leaves an adatom at 


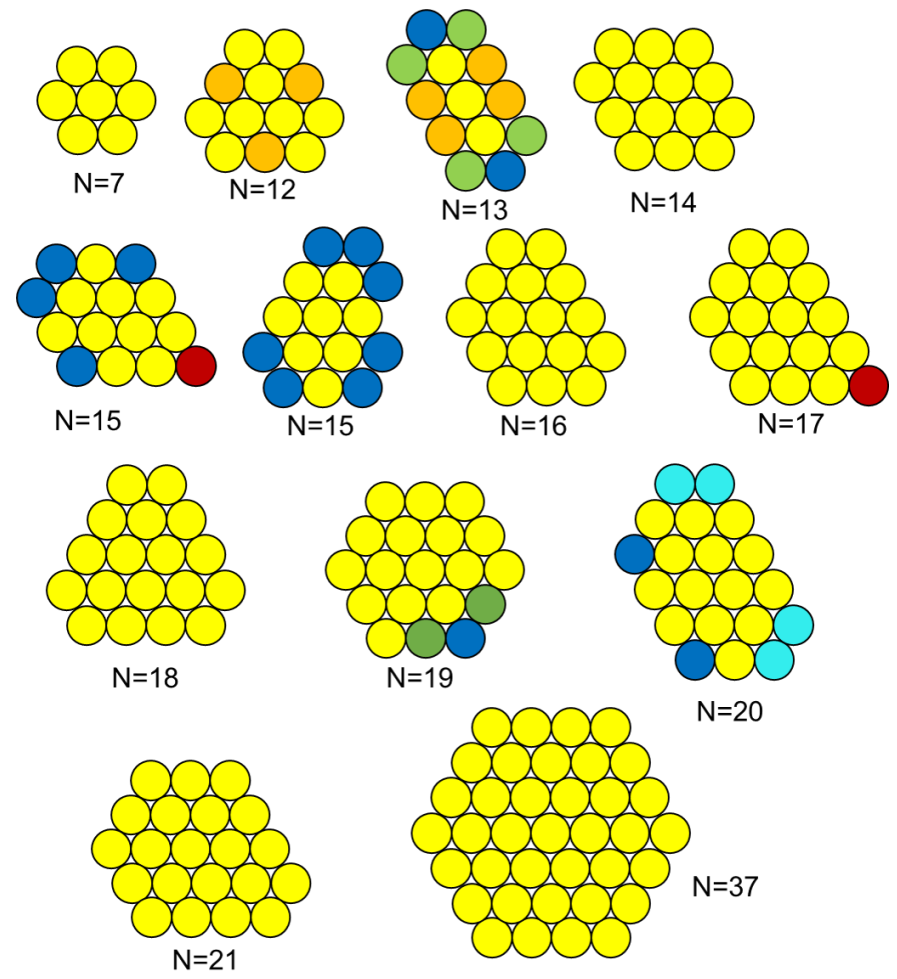

Figure 5: Low-energy shapes of clusters with a fixed number of atoms. Clusters with a compact, and thus very likely, shape can be drawn for different sizes, not only for $\mathrm{N}=7,19$ and 37 (that have compact, 6-fold symmetric shape). Blue and green atoms are kinks, with 3 in-plane neighbors; orange atoms (belonging to edges) have 4 in-plane neighbors; the red atom (adatom at a step) has only 2 in-plane neighbors. For $\mathrm{N}=20$ coupled kinks are light blue, normal kinks are dark blue.

step, with only 2 in-plane neighbors, easier to detach. $\mathrm{Nu}-$ cleation of a facet is thus easier for $\mathrm{N}=20$ and $D_{C}^{20}>D_{C}^{19}$. For $\mathrm{N}=21$, the compact shape has only 2 coupled kinks, less than $\mathrm{N}=20$, therefore the probability to remove one of the coupled kinks (and to leave one adatom at step with 2 in-plane neighbors) is lower than for $\mathrm{N}=20$, so $D_{C}^{20}>D_{C}^{21}$. The compact configurations with $\mathrm{N}=12$ and $\mathrm{N}=13$ have both 6 coupled kinks. However for $\mathrm{N}=13$ two of the kinks are special because removing one of them (for instance the blue one in figure 5 leaves two adatoms at step (green in figure 5 instead of one. Therefore $D_{C}^{13}>D_{C}^{12}$. Only two coupled kinks are present for $\mathrm{N}=14$, so $D_{C}^{14}<D_{C}^{13}$. Similar reasoning can explain all the other oscillations of $D_{C}$ in the red boxes of figure 4. No compact shapes with only 6 kinks can be found for clusters with $\mathrm{N}=15$ and $\mathrm{N}=17$, they have either one adatom at step on the boundary or a shape with 7 kinks. These two clusters should emit the adatom at step very easily to show a compact shape, and this atom should help the nucleation of new steps and thus should make the island displace faster than the island with size N-1. However this is not observed in the value of $D_{C}$, i.e. no significant oscillations of $D_{C}$ are observed between 14 and 17. The increase of $D_{C}$ at $\mathrm{N}=18$ is due to the 6 coupled kinks of the cluster compact shape.
It should also be considered that low energy configurations can have, for different sizes $\mathrm{N}$, different number of atoms constituting the steps. A configuration with more step atoms has more probability to emit a boundary atom, and thus to make the cluster move, than a configuration with less step atoms. This effect could add to the "coupled kinks" effect explained in the previous paragraph. For instance, the compact, low energy configuration of the cluster with $\mathrm{N}=12$ has 3 step atoms (highlighted in orange in figure 5); a cluster with $\mathrm{N}=13$ is larger and should thus have lower $D_{C}$, but its compact configuration has 4 step atoms and can thus account for $D_{C}^{13}>D_{C}^{12}$. The change of number of step atoms in compact shapes explain almost all the observed oscillations. An exception is the compact configuration of the cluster with $\mathrm{N}=14$, that has the same number of step atoms of the cluster with $\mathrm{N}=13$. Thus the large decrease of $D_{C}$ between $\mathrm{N}=13$ and $\mathrm{N}=14$ is due to the general decrease of $D_{C}$ with the size and to the "coupled kinks" effect.
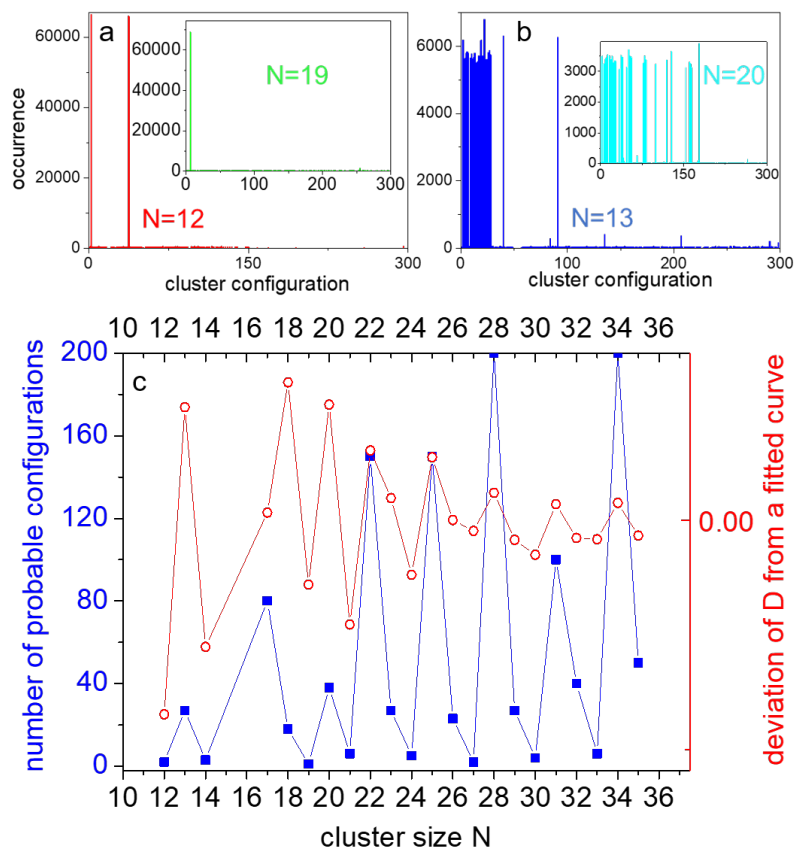

Figure 6: a and b: Histograms of the occurrence of different configurations for clusters with $\mathrm{N}=12$ and $\mathrm{N}=13$. The insets show the histograms for $\mathrm{N}=19$ and $\mathrm{N}=20$. c: The red circles show the deviation of $D_{C}$ from a curve fit of the $D_{C}$ data in figure 4 . The aim of these points is to highlight the oscillations of $D_{C}$ due to the finite-size effect. The blue squares represent the number of probable configurations for each cluster dimension.

Another way to discuss the oscillations is to consider the number of possible configurations that can be taken by a diffusing cluster. This number increases with the cluster size, but, for fixed $\mathrm{N}$, some configurations have lower energy and are thus more probable than others. For each cluster with size $\mathrm{N}$, we have run simulations to allow for the rearrangement of a cluster shape. During the simulation, at fixed intervals, the cluster configuration was compared 
with those already found. At the end of the simulation, we have counted the number of times all the different cluster configurations appears. Figures $6 \mathrm{a}$ and $6 \mathrm{~b}$ show two histograms of the occurrence of the different configurations for the cluster with size $\mathrm{N}=12$ and $\mathrm{N}=13$ (red and dark blue bars respectively). The cluster with $\mathrm{N}=12$ tends to have 2 different configurations, while others are much less probable. The island with $\mathrm{N}=13$ can have many different configurations $(\approx 30)$, with similar probability, while other configurations are less probable. It can thus be assumed that, for the cluster with size $\mathrm{N}=12$, atoms detach but often re-attach back at the same position, leading to few probable cluster configurations and delaying cluster diffusion. The island with $\mathrm{N}=13$ can have different configurations with similar probability and should thus diffuse faster than that with $N=12$. The insets in figure 6 and 6 b show another example of configurations found for clusters with $\mathrm{N}=19$ and $\mathrm{N}=20$. As expected, the cluster with $\mathrm{N}=19$ has very often the configuration corresponding to the symmetric perfect size (see figure 5). Figures 6c shows the number of most probable configurations as a function of the cluster size, together with the diffusivity corrected of the monotonic descending trend. A configuration $\gamma$ is considered probable if it is found more than 100 times with respect to a low probable configuration and if there are no other configurations that occur 5 times more than $\gamma$. The clusters with few probable configurations, i.e. those that tend to show very often the same shape, have lower diffusivity. An exception, addressed above considering the number of step atoms in the compact shape, is for $\mathrm{N}=17$ and 18. For clusters larger than $\mathrm{N}=35$, the oscillations are within the error of the measured diffusion coefficient. The number of configurations is also an argument evoked on square lattices [9. The main difference is that, as hexagonal islands have higher symmetry than square ones, the oscillations in figure 4 have only weak amplitude and they disappear for $\mathrm{N}>35$, that is a size much smaller than that observed for square lattice ( $N=200 / 300)$. The perfect size effect, as also highlighted by Heinonen et al. [27], is only observed at low temperatures, where the difference of Boltzmann factor among the different atomic jumps is large.

\subsection{Finite-size effects: 2D voids}

Like islands, also voids show a finite-size dependence of the diffusion coefficient from the size. Figure 7 shows $D_{C}$ as a function of the size of voids. Clusters with small size often splits during the long simulations necessary to average the cluster squared displacement, and are thus not considered. The general trend is an increase of $D_{C}$ with the size up to about $\mathrm{N}=50$, where a plateau is found. Then $D_{C}$ decreases at first slowly and, for $\mathrm{N}>300$, steadily, with $D_{C} \propto N^{-0.65 \pm 0.05}$. Notice that $N^{-0.65}$ corresponds to $R^{-1.3}$ found in the cluster-diffusion section. Most of the data, within the simulation error, follow a continuous curve. However, as in the case of islands, there are some clear deviations of $D_{C}$ from the general trend, particularly for $\mathrm{N}=7,10$ and 19 . The increase of $D_{C}$ with the size for

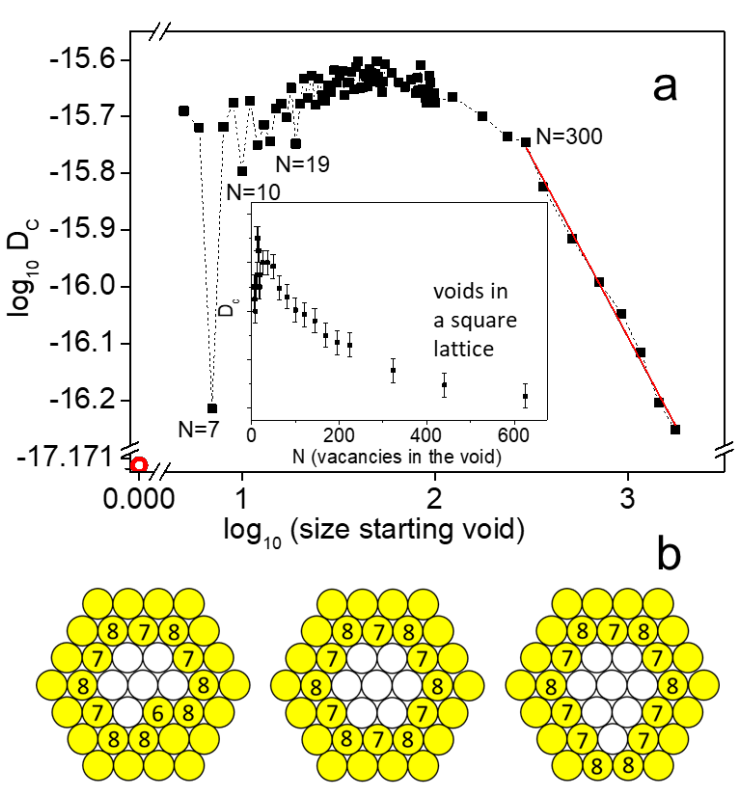

Figure 7: The dark squares show the cluster diffusion coefficient $D_{C}$ as a function of the cluster size $\mathrm{N}$ (number of empty positions) in $\log -\log$ scale. The diffusion coefficient of a vacancy is also shown as a red circle. Notice that both the $\mathrm{x}$ and the y axis have a break. $D_{C}$ generally increases with the cluster size up to a plateau around $\mathrm{N}=50$ and then decreases. Certain sizes show significant deviation from the general trend. Three compact configurations for voids with $\mathrm{N}=6,7$ and 8 are shown in the bottom of the figure. The boundary atoms (yellow) have different jump energies (values written in $E_{b}$ units).

small $\mathrm{N}$ is surprising, was not observed for islands, was not investigated by other authors and thus deserves a discussion. As in the case of single adatoms, $D_{C}=\frac{1}{4} \Gamma_{C} \cdot \lambda_{C}^{2}$, where $\Gamma_{C}$ is the cluster jump frequency, that depends on the sum of the detachment rates of boundary atoms and on the diffusion time of adatoms inside the voids; $\lambda_{C}$ is the jump length, that decreases slowly when the void size increases. Increasing the void size, the number of boundary atoms that can move increases and their jump rates also increase. For instance, the motion of an isolated vacancy $\left(D_{C}\right.$ marked with a red circle in figure $7 a$ ) depends on the jump rates of the 6 atoms around it, $\propto \exp \frac{-8 E_{b}}{k T}$. The "perfect" shape of a void with $\mathrm{N}=7$, that should be very stable, has 6 boundary atoms with jump rate $\exp \frac{-8 E_{b}}{k T}$ and 6 other border atoms with jump rates $2 \exp \frac{-7 E_{b}}{k T}$. It thus displaces faster than the isolated vacancy. The sum of the detachment rates of boundary atoms in clusters with nonperfect shape is even higher than that of the void with $\mathrm{N}=7$ and thus they move faster. For instance the void with $\mathrm{N}=6$ in figure $7 \mathrm{p}$ has a boundary atom with high detachment rate $\left(3 \exp \frac{-6 E_{b}}{k T}\right)$, and the void with $\mathrm{N}=8$ has seven boundary atoms (instead of six for the cluster $\mathrm{N}=7$ ) with detachment rate $2 \exp \frac{-7 E_{b}}{k T}$. For small voids the diffusion time inside the voids is not significant and thus $\Gamma_{C}$ increases with the size. Increasing the void size, the cluster jump length decreases and the diffusion time of the adatoms in- 
side the void increases, reducing $\Gamma_{C}$. Therefore for large voids $D_{C}$ decreases when the size increases. For comparison, in very small islands, the jump rates of border atoms decrease when the island size increases (for instance an adatom has high jump frequency, $\exp \frac{-3 E_{b}}{k T}$, while atoms in a cluster are more strongly bound and have smaller jump rates, $\propto \exp \frac{-4 E_{b}}{k T}$ or smaller). Furthermore, the diffusion time outside islands before attachment is high for small islands and decreases when the island size increases (increased cross section and thus increased probability of adatom attachment). Therefore $\Gamma_{C}$ (and thus $D_{C}$ ) always decreases when the island size increases.

The behavior described above concerning the diffusivity of voids with different sizes is not typical of hexagonal lattices: In preliminary simulations on a square lattice we have observed the same features (see the inset of figure 7 a).

\section{Displacement under an external force}

\subsection{Single atoms/vacancies}
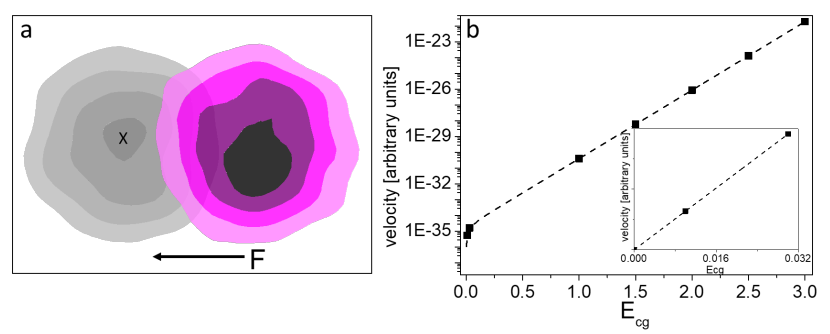

Figure 8: a: Distribution of the final positions of a diffusing vacancy, after 500 simulations. The grey and purple areas correspond to $E_{c g}=0$, and 0.03 respectively. The purple and grey probability distributions are partially overlapped. The starting position of the vacancy is the same for the two sets of simulations and marked by X. b: Velocity of a vacancy for different force (and thus $E_{c g}$ ) values. The y axis is in logarithmic scale, the velocity depends exponentially from $E_{c g}$. The inset shows that for small $E_{c g}$ the vacancy velocity depends linearly from the force. $\mathrm{kT}=0.1 E_{b}$.

Applying a force, the diffusion of single atoms and vacancies is biased in the force direction. The grey area in figure 8 a shows the probability distribution of the final position of a vacancy starting from the point marked by $\mathrm{X}$ and diffusing (without force, $E_{c g}=0$ ) for a fixed time. The figure is based on 500 simulations, a darker color corresponds to higher probability. Applying a weak force on the atoms towards the left, the vacancy does not diffuse anymore completely randomly around the starting position but displaces preferentially towards the right. This is shown by the purple probability distributions of the final positions of a vacancy for 500 simulations performed with $E_{c g}=0.03$. The average final position of the vacancy is displaced towards the right, proportionally to the applied force. For strong forces the distribution is less dispersed, the vacancy final position is well defined, with only a small deviation from the most probable position. Similar results are obtained for single atoms, the main difference is that they displace in the same direction of the force and the jump energy is lower ( $3 E_{b}$ instead of $8 E_{b}$ for vacancies). Considering the time elapsed in the simulations, the vacancy velocity can be measured and is shown in figure $8 \mathrm{~b}$ as a function of the force. When a force affects a jump, the jump frequencies are modified and the velocity $v$ against the force direction is equal to the difference between the jump frequencies along and against the force of the atoms surrounding the vacancy. Considering the 6 possible jumps of the vacancy:

$$
\begin{array}{r}
v=a \cdot\left(\exp \left(-\frac{E_{v a c}-E_{c g}}{k T}\right)+\frac{1}{2} \exp \left(-\frac{E_{v a c}-0.5 E_{c g}}{k T}\right)\right. \\
-\frac{1}{2} \exp \left(-\frac{E_{v a c}+0.5 E_{c g}}{k T}\right)-\exp \left(-\frac{E_{v a c}+E_{c g}}{k T}\right) \\
\left.-\frac{1}{2} \exp \left(-\frac{E_{v a c}+0.5 E_{c g}}{k T}\right)+\frac{1}{2} \exp \left(-\frac{E_{v a c}-0.5 E_{c g}}{k T}\right)\right) \\
=a \cdot \exp \left(-\frac{E_{v a c}}{k T}\right) \cdot\left(\exp \left(\frac{E_{c g}}{k T}\right)-\exp \left(-\frac{E_{c g}}{k T}\right)\right. \\
\left.+\exp \left(\frac{0.5 E_{c g}}{k T}\right)-\exp \left(-\frac{0.5 E_{c g}}{k T}\right)\right)
\end{array}
$$

The velocity as a function of the force found in the simulations agree perfectly with the equation above, as shown by the fit of the dashed line with the black squares in figure 8p. For small forces (and thus small $E_{c g}$ ), $\exp x=1+x$ and therefore

$$
v=3 a \frac{E_{c g}}{k T} \cdot \exp \left(-\frac{E_{v a c}}{k T}\right)=3 a^{2} \frac{F}{2 k T} \cdot \exp \left(-\frac{E_{v a c}}{k T}\right)
$$

because, as shown in figure 2 the energy $E_{c g}$ is related to the force acting on the atoms as $F \cdot \frac{a}{2}=E_{c g}$. As detailed in the supplementary material, where we study the diffusion of single atoms and single vacancies, $D_{v a c}=$ $\frac{3}{2} \cdot a^{2} \cdot \exp \left(-\frac{E_{v a c}}{k T}\right)$. Therefore,

$$
v=\frac{F \cdot D_{v a c}}{k T}
$$

This expression corresponds to the Nernst-Einstein relation.

\subsection{Clusters}

Under the effect of a force, as a consequence of atomic displacement, clusters also move. This motion can be limited by diffusion of atoms at the cluster periphery, by diffusion on the terrace, and by attachment/detachment kinetics (this last mechanism becomes important if diffusion is easy and fast with respect to the detachment). Within our model all these mechanisms can take place, and the balance among them depends on parameters like the Boltzmann factor and the size of the islands. As atomic jumps depend on the number of neighbors of the starting position and not on that of the final state (we use KMC in Arrhenius dynamics [12] and not Metropolis Monte Carlo), periphery diffusion is not particularly favored, contrary to other works where atoms are constrained to move only at 


\section{terrace diffusion / attachment-detachment}
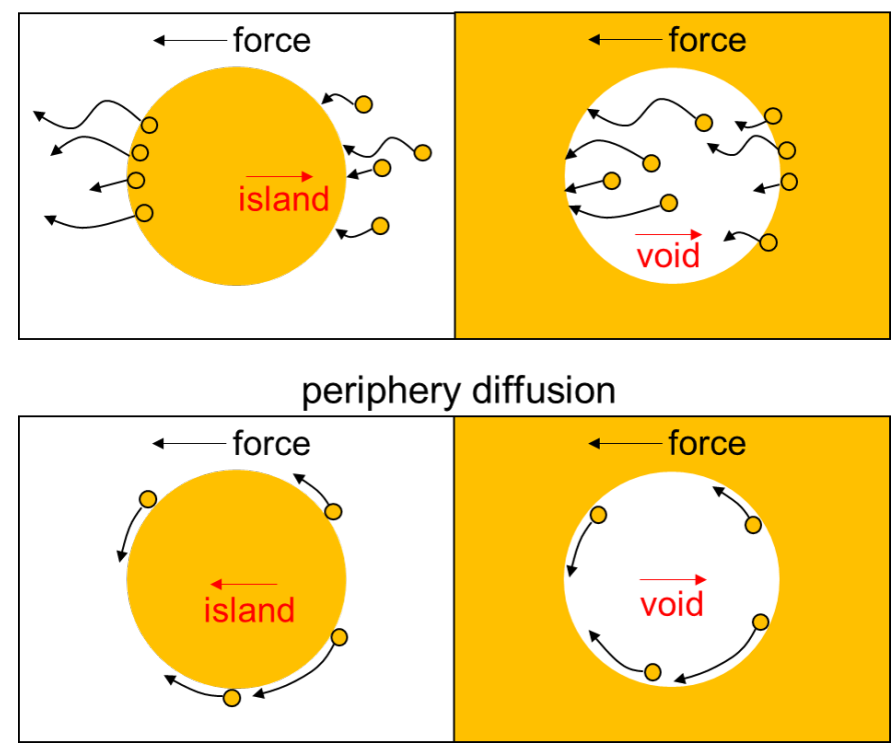

Figure 9: Schematics of the motion of $2 \mathrm{D}$ islands and voids as a consequence of forced diffusion. $2 \mathrm{D}$ voids always move opposite to the force acting on the adatoms, while $2 \mathrm{D}$ islands can move in the force direction when the motion is determined by periphery diffusion.

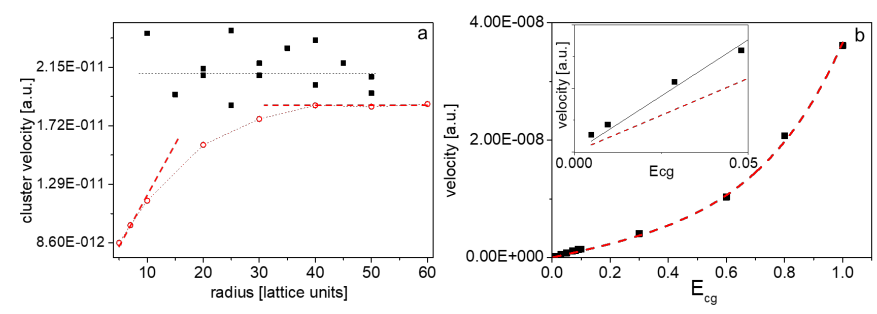

Figure 10: a: Velocity of islands (black squares) and voids (red empty circles) displacing under the effect of a force, as a function of the radius. $E_{c g}=0.001, \mathrm{kT}=0.3$. b: Velocity of an island $(\mathrm{R}=20)$ displacing in the direction opposite to the force, as a function of the force. The inset is a magnification of the main figure at low forces.

the cluster periphery (as for instance done in [5] in the section on periphery diffusion).

While in our model the atoms diffuse preferentially in the force direction, both islands and voids displace opposite to the force direction. As explained in $[5$ and summarized in figure 9 this occurs because in our simulations periphery diffusion does not play an important role. Clusters displace with constant velocity under the effect of a force. The velocity of islands and voids as a function of their size is shown in figure $10 \mathrm{a}$. While the velocity of islands does not depend on their size, that of voids is proportional to the cluster size for small sizes, and becomes constant after a threshold. According to [5], if the velocity of clusters moving under a force does not depend on their size, then the process limiting the displacement is terrace diffusion, while if the velocity is directly proportional to the size, then attachment and detachment processes play the most important role. Our findings for clusters subject to a force are thus consistent with those discussed in section 3, where we showed that for islands and voids the displacement is diffusion limited, and for small voids also the detachment kinetics plays an important role. Furthermore, the velocity of voids increasing with the void size and reaching a constant value correctly reproduces the experimental observations of $2 \mathrm{D}$ voids displacing under electromigration on $\operatorname{Si}(111)$ [15.

As shown in figure $10 \mathrm{p}$, for a fixed size the cluster velocity increases linearly with the force for small forces and follows an exponential law for strong forces. Supposing that the equation derived for vacancies in section 4.1 (and also valid for adatoms) can be used for clusters, we can fit the data to obtain $E_{\text {cluster }}$ (instead of $E_{v a c}$ ). We find $E_{\text {cluster }}=6.19 \pm 0.02 E_{b}$ (for $k T=0.3 E_{b}$ ) that is close to the diffusion energy of a cluster obtained in section 3 without forces $\left(E_{\text {cluster }}=6.06 \pm 0.05 E_{b}\right)$. The agreement is very good, the slight difference is probably due to the clusters stability (they have different shapes for different forces, see section 5.2). For weaker forces, where the cluster shape changes marginally, a fit with $E_{\text {cluster }}=6.06$ reproduces well the data (see the black line in the inset of figure $10 p$ ).

\section{Cluster shapes in a lattice with 3-fold symmetry}

The shape of clusters fluctuates around an equilibrium shape that depends on the temperature, on the binding energy and on the external force applied. For hexagonal clusters with 6 -fold symmetry, when $k_{n s l}=0$, the shape changes have been shown in [11. Here, we discuss the shapes of clusters on a lattice with 3 -fold symmetry.

\subsection{Cluster shape dependence on temperature}

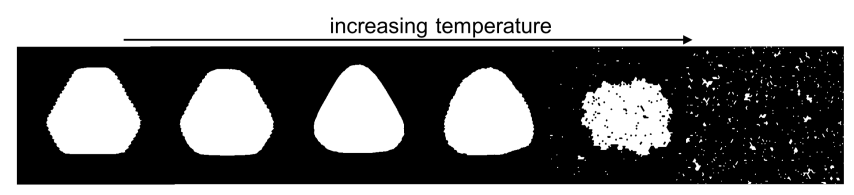

Figure 11: Shapes of a stationary 2D island (white) on a (111) surface at different temperatures. At low temperatures the images shown correspond to the average of several shape configurations.

Figure 11 shows the shape of an island at different temperatures, for $k_{n s l}=0.18$ (a parameter defining the relative stability of A and B steps, see appendix A). For each temperature, the simulations start from a round cluster; then atomic jumps are allowed, so that the shape rearranges; when the shape does not significantly evolve anymore, an average shape can be calculated. At low temperature corner and edges of the island are well defined; increasing the temperature the corners become rounder and the length of less stable edges decreases; at higher temperature the whole cluster is roundish, and straight edges 
are no more observed; at very high temperatures the cluster disintegrates in dispersed atoms. A similar behavior is observed for $2 \mathrm{D}$ voids.

\subsection{Shapes of clusters under strong forces}
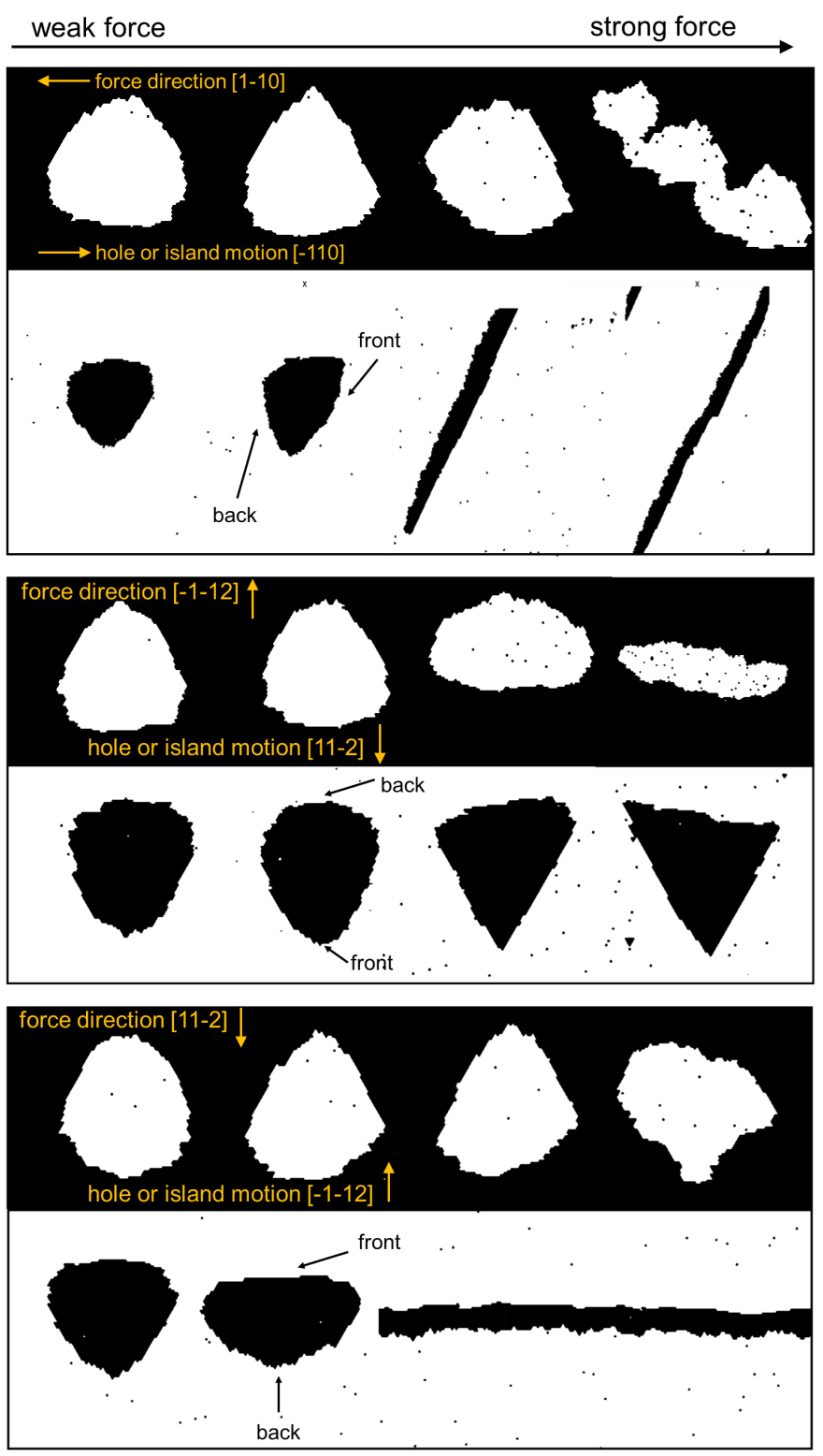

Figure 12: Shapes of a 2D island (first line) and a 2D hole (second line) on a (111) surface under a force that adds a bias to diffusion. The force is towards the left, the top and the bottom of the images for the three panels respectively. The force acting on the shapes implies an energy change from left to right $E_{c g}=0.0001,0.001,0.01$, $0.1 E_{b} . \mathrm{kT}=0.1 E_{b}$.

In absence of any force, clusters are at thermodynamic equilibrium with an adatom gas and their shape fluctuates around an equilibrium shape. Under external forces, a flux of adatoms leads to the cluster motion. Clusters are thus no more at equilibrium. Their shape can reach a steady state, or can fluctuate among different configurations. Kuhn, Krug, Rusanen and Dasgupta et al. have carefully studied this behavior with analytical models 36-40. We have studied the shapes of clusters moving under strong forces $\left(E_{c g}\right.$ larger than $10^{-4} E_{b}$ ), however we have not carefully investigated possible fluctuations between different configurations. Notice that average forces found in electromigration phenomena are of the order of $10^{-6} \mathrm{eV} /$ lattice length 6, that corresponds to $E_{c g} \approx 10^{-6} E_{b}$, with $E_{b}$ of the order of $1 \mathrm{eV}$. The 6 -fold symmetry is broken when the simulations are performed with $k_{n s l} \neq 0$ and the shape of holes and islands has a three-fold symmetry (see figure 11). We have studied the effects of forces applied in three different directions on the nanostructure shapes. Figure 12 shows snapshots of the simulations of islands and holes moving under the effect of these forces. Each snapshot corresponds to a representative island or void shape moving under a constant force. We consider that a cluster has reached a steady state shape when three shape descriptors do not significantly evolve anymore, as detailed in the supplementary material. We at first discuss the shapes of voids. When the force is towards the [1-10] direction (towards left in figure 12. first panel, second line), the shapes seem pushed towards the facet of the right, i.e. towards the advancing edge. The advancing edge is well faceted and its length increases with the force value. For very strong forces the voids are elongated and flattened on the right facet. Loosely bound and kink atoms are easily removed from the front under the effect of the force. The unzipping of the atomic rows by removal of the kinks (detachment of a kink atom leaves another kink atom that will also be detached) leads to a displacement of the front edge. This unzipping is only stopped at the corners of the cluster. The clusters tend to keep a convex shape to maximize the total number of bonds between atoms. However the force leads to elongation of the advancing edge and thus of the whole shape, because also atoms that do not belong to the front edge can detach. When this happens, atomic rows of that edge unzip; when the unzipping reach the front edge, this results elongated of one unit length towards the edge that has unzipped. Because of the force towards the back, the detached atoms accumulate at the back edge and only rarely they move against the force, towards the front edge. The cluster thus keep an elongated front edge because of a kinetic effect. A similar elongation, perpendicular to the motion, is observed when the force is in the [11-2] direction (third panel in figure 12). In this case the elongation is along the top facet, that corresponds to the advancing edge. Applying a force in the [-1-12] direction (second panel in figure 12), the elongation perpendicular to the motion is no more observed. Because of the triangular symmetry, an edge at the advancing front of the void would correspond to a A step, less stable than B steps. Under the effect of the force, atoms are removed more easily from A steps than from B steps. The lateral facets of the void (B steps) in the figure are thus enhanced. The void moves by unzipping of the atomic rows from the lateral facets as 
previously explained, the detached atoms diffuse towards the back edge where they accumulate, and the void shows a strongly faceted triangular shape. Latz et al. [41] simulated the shape of 3D holes in $\mathrm{Ag}(111)$. They found elongation of the holes along the force axis and formation of a tip at the hole advancing front. The difference of their results with ours is due to the different mechanism of atomic motion: in their simulations atoms diffuse along the edges, by periphery diffusion, while in our case atoms diffuse inside the hole, by terrace diffusion.

For islands the mechanisms are similar to those described for voids, but in this case atoms are removed from the back edge and accumulate to the front edge that thus advances. Under very strong forces, islands can also break down in smaller islands that advance together. While in voids atoms arriving at the back are stuck because of the force, in islands the atoms arriving to the front are pushed by the force towards the back moving along the periphery of the cluster. The less stable facets decrease in size or are rough, because atoms can be removed easily from them by the force. The most stable facets tend to be flattened because of the unzipping of atomic rows previously discussed for voids. Like voids, also islands tend to elongate along the most stable facets of the front edge. However, increasing the temperature, i.e. decreasing the step energy anisotropy, the island shape tends to elongate parallel to the motion, as shown in figure 13 for a triangular islands moving in the [-1-12] direction at three different temperatures. The elongation parallel to the force is more difficult for islands with the front facet perpendicular to the motion direction (those of the second panel in figure 12 and it is never observed for voids. The increased temperature decreases the anisotropy of the cluster shape, the tendency to form facets decreases and atoms also diffuse in the force direction along the island periphery, that gives a shape elongated in the force direction. In voids, the atoms do not have to displace along the void edges to follow the force direction and thus the elongation due to a periphery diffusion effect is absent.

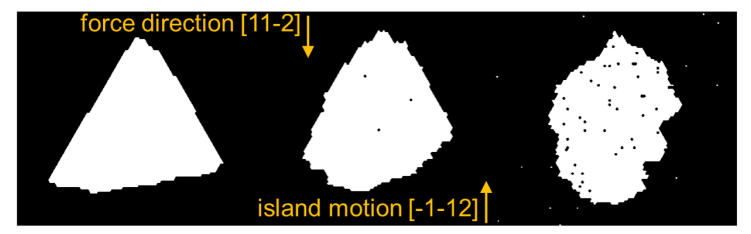

Figure 13: Shapes of a 2D island on a hexagonal lattice under a force implying an energy change $E_{c g}=0.01 E_{b}$ to the atomic jumps, that adds a bias to the atomic motion in the [11-2] directions. The temperatures are $\mathrm{kT}=0.05,0.1$ and $0.15 E_{b}$ for the shapes from left to right.

\section{Conclusions}

We have used a KMC model to highlight the diffusion behaviors of voids and islands in a hexagonal lattice. The diffusivity of small clusters does not depend on the size in a continuous way and a perfect size effect is clearly observed. Most of the results obtained on square lattices are also valid on hexagonal lattices, however subtle changes are evidenced. For instance, since hexagonal islands have higher symmetry than square ones, the oscillations of the diffusion coefficient that exist for the smallest sizes are weakened in hexagonal lattices. We also evidence the peculiar behavior of the diffusion coefficient of small voids: when the void size increases, the diffusivity at first increases then reaches a plateau and finally decreases. We show that this behavior is not specific to hexagonal lattices but also exists (but to the best of our knowledge has not yet been described) on square lattices. We also show how electromigration adds a bias to the atomic jumps, how the cluster velocity depends on the force strength and on the cluster size and how the cluster shapes change with the temperature and the force direction. Finally, we establish approximated analytical expressions of the diffusion coefficient of clusters that gives values in quite good agreement with the values extracted from the KMC simulations. These developments lead to a better understanding of the atomic events at the origin of the behavior of diffusing clusters and thus to the capability of controlling the motion of nano-objects on a surface. 


\section{Acknowledgments}

We thank B. Ranguelov and M. Michailov for helpful suggestions. We also thank A. Saul and O. Pierre-Louis for stimulating discussions. We acknowledge financial support from the French Region Sud Provence-Alpes-Cote d'Azur (MELOPEE project).

Declarations of interest: none

\section{Appendix A. Model details}

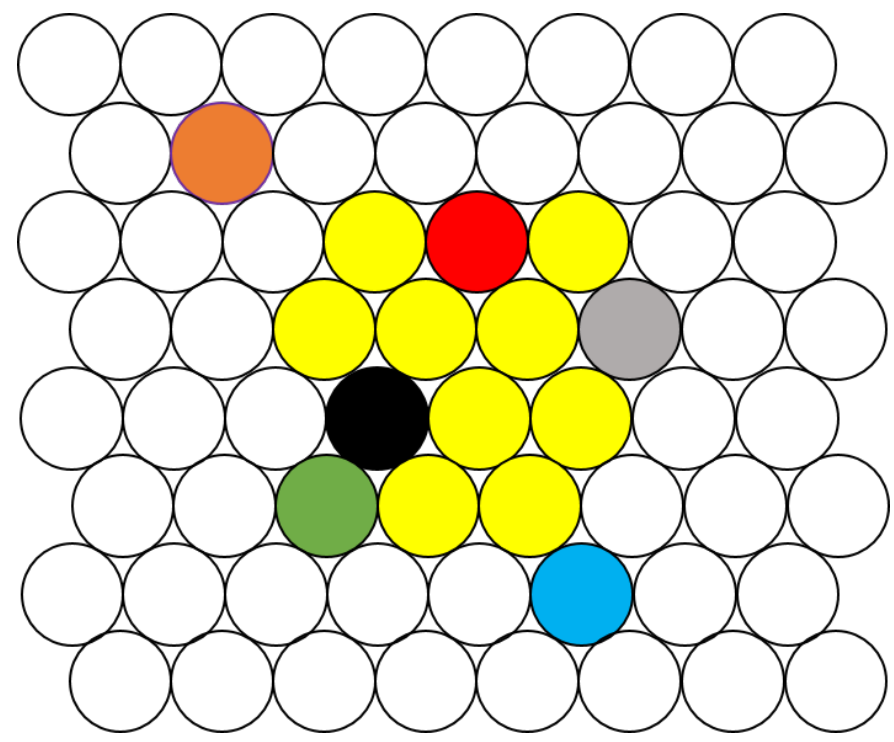

Figure A.14: The atoms at an island edge can have $\mathrm{j}=1$ (blue), 2 (green), 3(grey), 4(red) or 5(black) in-plane neighbors. Within our model, when $k_{n s l}=0$, their binding energy is respectively $4,5,6,7$ and $8 E_{b}$. An isolated adatom is shown in orange (binding energy $\left.3 E_{b}\right)$. Other atoms of the island are yellow, while empty positions are white.

This appendix completes the details of the model used in the simulations. In particular we explain the binding energy of the different kind of atoms considered. Every surface atom has three neighbors in the subsurface layer $\left(n s l_{1}, n s l_{2}, n s l_{3}\right)$ (see figure 1b). For each $n s l$ we count the number of neighbors $\left(n n_{n s l}\right)$ in the surface, value variable between 1 and 3 . Thus, $n n$ is variable between 3 (for an isolated adatom with 3 nearest neighbors in the underlying layer) and 9 (surface atoms surrounded by 6 in-plane nearest neighbors and 3 atoms in the underlying layer). The binding energy of each surface atom is:

$$
\begin{aligned}
E_{b i n}=E_{b} \cdot\left(n n_{n s l_{1}}-k_{n s l} \cdot n n_{n s l_{1}}^{2}\right. & \\
+n n_{n s l_{2}} & -k_{n s l} \cdot n n_{n s l_{2}}^{2} \\
& \left.+n n_{n s l_{3}}-k_{n s l} \cdot n n_{n s l_{3}}^{2}\right)
\end{aligned}
$$

Where $k_{n s l}$ is a parameter to be adjusted. This expression allows to differentiate the energy of atoms according to their environment and in particular reproduce the difference between $\mathrm{A}$ and $\mathrm{B}$ steps. When $k_{n s l}$ is 0 , atoms at steps $\mathrm{A}$ and $\mathrm{B}$ have the same binding energy and the clusters have hexagonal shape. In the text, energy values are expressed as multiples of $E_{b}$, for instance an isolated adatom, orange in figure A.14 has $E_{b i n}=3 E_{b}$. Atoms in kink positions, grey in figure A.14 have $E_{b i n}=6 E_{b}$. Atoms surrounding a vacancy have $E_{b i n}=8 E_{b}$ (values given for $k_{n s l}=0$ ). In the simulations, with the aim to reproduce general behaviors of clusters diffusing on a hexagonal lattice, we have used $E_{b}=1(\mathrm{eV})$. For specific systems, this value could be changed. For instance, for $\mathrm{Au}$, a value of $\mathrm{Eb}=0.64 \mathrm{eV}$ (see [42]) could be used. However, as the model is very simple, it cannot be used for accurate quantitative predictions. To obtain shapes with a three fold symmetry (like on $\operatorname{Pt}(111)$ [13 or $\operatorname{Si}(111)$ [4] ) we have used $k_{n s l}$ values between 0.05 and 0.18 . The ratio between the binding energies of atoms at B and A steps results less than 0.9 , a reasonable value for (111) fcc and diamond systems 13,44 .

\section{Appendix B. Cluster energy}

Expression 2 used in section 3.1 does not directly lead to $E_{\text {cluster }}=E_{\text {kink }}$ but can be useful to further discuss our results. Clusters tend to be compact, and the boundary atoms have $\mathrm{j}=2\left(E=5 E_{b}\right.$, atoms adsorbed at steps, green in figure A.14), $\mathrm{j}=3$ ( $E=6 E_{b}$, kink atoms, grey in figure A.14 or $\mathrm{j}=4$ ( $E=7 E_{b}$, step atoms, red in figure A.14). The cluster energy we find $\left(\approx 6 E_{b}\right)$ does not correspond to the energy of atoms adsorbed at the cluster steps $\left(5 E_{b}\right)$ because the displacement of these atoms implies only a fluctuation of the position of the cluster around a certain position. An important displacement of the cluster can instead be obtained when kinks are removed $\left(E_{j}=6 E_{b}\right)$, as they lead to the unzipping of entire atomic rows. The boundary atoms constituting the cluster edges $(\mathrm{j}=4)$ are many but they are strongly bound $\left(7 E_{b}\right)$ and, because of the exponential term in the concentration expression, their contribution to the adatom concentration or to the effective cluster energy is small. However in voids, where the boundary is concave, they could play a role and could explain why the cluster energy found in the simulation is slightly higher than the kink energy.

In order to check a practical example, we have counted the different $N_{j}$ of a cluster with $\mathrm{R}=10$ at $\mathrm{kT}=0.35$. Averaged over many cluster configurations, $N_{2}=2 \quad N_{3}=18$ $N_{4}=32$. With these values, we find

$$
<\exp \left(\frac{-E_{j}}{k T}\right)>=\exp \left(-6 E_{b} / k T\right)
$$

where $6 E_{b}$ corresponds to the value of $E_{C l u s t e r}$ that we have found. 


\begin{tabular}{|c|c|}
\hline$k_{n s l}$ & parameter defining the three fold symmetry \\
\hline$E_{\text {bin }}$ & binding energy of an atom, depending on the atom environment \\
\hline$E_{b}$ & energy unit in our model \\
\hline$E_{c g}$ & change of the jump energy barrier due to the force \\
\hline$E_{\text {kink }}$ & binding energy of an atom in kink position \\
\hline$E_{a d-s t e p}$ & binding energy of an atom adsorbed at a step \\
\hline$k$ & Boltzmann constant \\
\hline$T$ & temperature \\
\hline$F$ & external force changing the jump energies \\
\hline$D_{a d}$ & adatom diffusion coefficient \\
\hline$E_{a d}$ & adatom jump/diffusion energy \\
\hline$a$ & adatom jump length \\
\hline$<r^{2}>$ & adatom average square displacement \\
\hline$<r_{\text {cluster }}^{2}>$ & cluster average square displacement \\
\hline$t$ & time \\
\hline$E_{v a c}$ & vacancy activation energy \\
\hline$D_{C}$ & cluster diffusivity \\
\hline$R$ & cluster radius \\
\hline$\alpha$ & exponent defining the atomic mechanism limiting the diffusion of clusters \\
\hline$\lambda_{C}$ & unit cluster displacement \\
\hline$\Gamma$ & jump frequency \\
\hline$\Omega$ & unit cell area \\
\hline$c_{l}$ & atomic linear concentration at the cluster edge \\
\hline$t_{\text {detachment }}$ & time necessary to detach an atom from a cluster edge \\
\hline$t_{\text {attachment }}$ & time for an attachment event \\
\hline$t_{a t-d i f}$ & adatom diffusion time before attachement to the cluster \\
\hline$c$ & adatom concentration \\
\hline$D_{0}$ & prefactor in the adatom diffusivity expression \\
\hline$D_{C 0}$ & prefactor in the cluster diffusivity expression \\
\hline$E_{\text {Cluster }}$ & cluster diffusion energy \\
\hline$N$ & number of atoms in the island or of empty positions in the void \\
\hline$N_{\text {edge }}$ & average number of atoms of a cluster edge \\
\hline$N_{\text {boundary }}$ & total number of atoms of a cluster perimeter \\
\hline$v$ & velocity of a vacancy displacing under the effect of a force \\
\hline$n s l_{1}, n s l_{2}, n s l_{3}$ & $\begin{array}{l}\text { indexes identifying three different atoms of the substrate that are neighbors of a } \\
\text { certain atom in the surface }\end{array}$ \\
\hline$n n_{n s l_{1}}$ & number of atoms in the surface that are neighbors of the substrate atom $n s l_{1}$ \\
\hline
\end{tabular}




\section{References}

[1] W. Burton, N. Cabrera, F. Frank, Anisotropic diffusion in stress fields, Philosophical Transactions of the Royal Society A243 (1951) 299.

[2] A. F. Voter, Classically exact overlayer dynamics: Diffusion of rhodium clusters on Rh(100), Physical Review B 34 (1986) 6819 .

[3] S. Curiotto, P. Müller, F. Cheynis, F. Leroy, 2D Manipulation of Nanoobjects by Perpendicular Electric Fields: Implications for Nanofabrication, ACS Applied Nano Materials 3 (2020) 1118.

[4] C. Tao, W. G. Cullen, E. D. Williams, Visualizing the electron scattering force in nanostructures, Science 328 (2010) 736.

[5] O. Pierre-Louis, T. L. Einstein, Electromigration of single-layer clusters, Physical Review B 62 (2000) 13697.

[6] S. Curiotto, P. Müller, A. El-Barraj, F. Cheynis, O. PierreLouis, F. Leroy, 2D nanostructure motion on anisotropic surfaces controlled by electromigration, Applied Surface Science 469 (2019) 463.

[7] F. Leroy, A. El-Barraj, F. Cheynis, P. Müller, S. Curiotto, Atomic Transport in Au-Ge Droplets: Brownian and Electromigration Dynamics, Physical Review Letters 123 (2019) 176101.

[8] S. V. Khare, T. L. Einstein, Brownian motion and shape fluctuations of single-layer adatom and vacancy clusters on surfaces: Theory and simulations, Physical Review B 54 (1996) 11752.

[9] K. C. Lai, J. W. Evans, D.-J. Liu, Diverse nanoscale cluster dynamics: Diffusion of 2D epitaxial clusters, The Journal of Chemical Physics 147 (2017) 201101

[10] K. C. Lai, D.-J. Liu, J. W. Evans, Diffusion of twodimensional epitaxial clusters on metal (100) surfaces: Facile versus nucleation-mediated behavior and their merging for larger sizes, Physical Review B 96 (2017) 235406.

[11] S. Curiotto, F. Leroy, P. Müller, F. Cheynis, M. Michailov, A. El-Barraj, B. Ranguelov, Shape changes of two-dimensional atomic islands and vacancy clusters diffusing on epitaxial (111) interfaces under the impact of an external force, Journal of Crystal Growth 520 (2019) 42.

[12] A. F. Voter, Radiation Effects in Solids, Springer, NATO Publishing Unit, Dordrecht, The Netherlands, 2005.

[13] T. Michely, G. Comsa, Visualizing the electron scattering force in nanostructures, Surface Science 256 (1991) 217.

[14] S. Rousset, V. Repain, G. Baudot, Y. Garreau, J. Lecoeur, Selfordering of $\mathrm{Au}(111)$ vicinal surfaces and application to nanostructure organized growth, Journal of Physics: Condensed Matter 15 (2003) S3363.

[15] F. Leroy, A. El-Barraj, F. Cheynis, P. Müller, S. Curiotto, Electric forces on a confined advacancy island, Physical Review B 102 (2020) 235412 .

[16] J.-M. Wen, S.-L. Chang, J. W. Burnett, J. W. Evans, P. A. Thiel, Diffusion of large two-dimensional Ag clusters on Ag(100), Physical Review Letters 73 (1994) 2591.

[17] K. Morgenstern, G. Rosenfeld, B. Poelsema, G. Comsa, Brownian motion of vacancy islands on $\mathrm{Ag}(111)$, Physical Review Letters 74 (1995) 2058.

[18] W. W. Pai, A. K. Swan, Z. Zhang, J. F. Wendelken, Island Diffusion and Coarsening on Metal (100) Surfaces, Physical Review Letters 79 (1997) 3210.

[19] D. C. Schlosser, K. Morgenstern, L. K. Verheij, G. Rosenfeld, F. Besenbacher, G. Comsa, Kinetics of island diffusion on $\mathrm{Cu}(111)$ and $\mathrm{Ag}(111)$ studied with variable-temperature STM, Surface Science 2000465 (2000) 19.

[20] C. D. Van Siclen, Single jump mechanisms for large cluster diffusion on metal surfaces, Physical Review Letters 75 (1995) 1574 .

[21] S. V. Khare, N. C. Bartelt, T. L. Einstein, Diffusion of Monolayer Adatom and Vacancy Clusters: Langevin Analysis and Monte Carlo Simulations of their Brownian Motion, Physical Review Letters 75 (1995) 2148.

[22] K. Binder, M. H. Kalos, "Critical Clusters" in a Supersaturated Vapor: Theory and Monte Carlo Simulation, Journal of Statistical Physics 22 (1980) 363.
[23] H. C. Kang, P. A. Thiel, J. W. Evans, Cluster diffusivity: structure, correlation, and scaling, The Journal of Chemical Physics 93 (1990) 9018 .

[24] D. S. Sholl, R. T. Skodje, Diffusion of clusters of atoms and vacancies on surfaces and the dynamics of diffusion-driven coarsening, Physical Review Letters 75 (1995) 3158.

[25] L. Bitar, P. Serena, P. Garcia-Mochales, N. Garcia, V. Thien Binh, Mechanism for diffusion of nanostructures and mesoscopic objects on surfaces, Surface Science 339 (1995) 221.

[26] A. Bogicevic, S. Liu, J. Jacobsen, B. Lundqvist, H. Metiu, Island migration caused by the motion of the atoms at the border: size and temperature dependence of the diffusion coefficient, Physical Review B 57 (1998) R9459.

[27] J. Heinonen, I. Koponen, J. Merikoski, T. Ala-Nissila, Island diffusion on metal fcc(100) surfaces, Physical Review Letters 82 (1999) 2733.

[28] R. Deak, Z. Neda, P. Barna, A Kinetic Monte Carlo Approach for Self-Diffusion of Pt Atom Clusters on a Pt(111) Surface, Communications of Computational Physics 10 (2011) 920.

[29] K. Morgenstern, E. Laegsgaard, F. Besenbacher, Motion of vacancy islands on an anisotropic surface: Theory and kinetic Monte Carlo simulations, Physical Review B 66 (2002) 115408.

[30] The size of the simulation box affects the number of adatoms in the box but does not change their concentration and should thus not play a role in $t_{\text {attachment }}$ if the concentration is homogeneous everywhere.

[31] G. Sitja, S. Le moal, M. Marsault, G. Hamm, F. Leroy, C. Henry, Transition from Molecule to Solid State: Reactivity of Supported Metal Clusters, Nano Letters 13 (2013) 1977.

[32] G. Sitja, H. Tissot, C. Henry, Particle size effect on the Langmuir-Hinshelwood barrier for $\mathrm{CO}$ oxidation on regular arrays of Pd clusters supported on ultrathin alumina films, Journal of Chemical Physics 151 (2019) 174703.

[33] G. L. Kellogg, Atomic view of cluster diffusion on metal surfaces, Progress in Surface Science 53 (1996) 217.

[34] Z.-P. Shi, Z. Zhang, A. K. Swan, J. F. Wendelken, Dimer shearing as a novel mechanism for cluster diffusion and dissociation on metal (100) surfaces, Physical Review Letters 76 (1996) 4927.

[35] P. Salo, J. Hirvonen, I. T. Koponen, O. S. Trushin, J. Heinonen, T. Ala-Nissila, Island migration caused by the motion of the atoms at the border: size and temperature dependence of the diffusion coefficient, Physical Review B 64 (2001) 161405(R).

[36] P. Kuhn, J. Krug, Islands in the stream: Electromigrationdriven shape evolution with crystal anisotropy, International Series of Numerical Mathematics 149 (2005) 159.

[37] J. Krug, Nonlinear Dynamics of Surface Steps, Wiley-VCH, Weinheim, Germany, 2010.

[38] M. Rusanen, P. Kuhn, J. Krug, Kinetic Monte Carlo simulations of oscillatory shape evolution for electromigration-driven islands, Physical Review B 74 (2006) 245423.

[39] D. Dasgupta, G. I. Sfyris, D. Maroudas, Current-driven morphological evolution of single-layer epitaxial islands on crystalline substrates, Surface Science 618 (2013) L1.

[40] D. Dasgupta, A. Kumar, D. Maroudas, Analysis of currentdriven oscillatory dynamics of single-layer homoepitaxial islands on crystalline conducting substrates, Surface Science 669 (2018) 25 .

[41] A. Latz, S. Sindermann, L. Brendel, G. Dumpich, F. J. Meyer zu Heringdorf, D. E. Wolf, Simulation of electromigration effects on voids in monocrystalline Ag films, Physical Review B 85 (2012) 035449.

[42] S. Curiotto, F. Cheynis, F. Leroy, P. Müller, Surface diffusion of $\mathrm{Au}$ on $\sqrt{3} \times \sqrt{3 \mathrm{Si}}(111)$-Au studied by nucleation-rate and Ostwald-ripening analysis, Surface Science 647 (2016) L8.

[43] N. Akutsu, A. Yasuhiro, Statistical mechanical calculation of anisotropic step stiffness of a two-dimensional hexagonal latticegas model with next-nearest-neighbour interactions: application to Si(111) surface, Journal of Physics: Condensed Matter 11 (1999) 6635.

[44] M.-C. Marinica, C. Barreteau, D. Spanjaard, M.-C. Desjon- 
quères, Diffusion Rates of $\mathrm{Cu}$ adatoms on $\mathrm{Cu}(111)$ in the presence of an adisland nucleated at fcc or hcp sites, Physical Review B 72 (2005) 115402. 